\title{
Distribution of mechanoreceptive sensilla and their functions in the defensive behavior of tenebrionid beetle pupae
}

This article was published in the following Dove Press journal:

Open Access Insect Physiology

20 May 2011

Number of times this article has been viewed

\author{
Toshiaki Kurauchi' \\ Tatsuya Nakamura' \\ Yoshihiro Toh ${ }^{2}$ \\ Toshio Ichikawa ${ }^{2}$ \\ 'Basic Biology, Graduate School of \\ System Life Sciences, ${ }^{2}$ Department of \\ Biology, Faculty of Sciences, Kyushu \\ University, Fukuoka, Japan
}

Background: Pupae of the tenebrionid beetles exhibit different defensive behaviors in response to tactile stimulation of different parts of their bodies. Little is known about the mechanosensory mechanisms that initiate these defensive behaviors. To understand the mechanisms, we examined the distribution and function of the mechanoreceptive sensilla on the pupal body of Zophobas atratus.

Methods: The sensilla on the surface of the pupal cuticle were observed using light and scanning electron microscopy. The tactile sensitivity of various parts of the pupal body was tested using three probes with different bending forces. The function of particular sensilla was examined by stimulating them electrically.

Results: Hair (trichoid) sensilla were classified according to their length, ie, long hair sensilla (80-200 $\mu \mathrm{m}$ ) were mostly located on the lateral regions of each body segment and short hairs $(5-50 \mu \mathrm{m})$ were distributed across almost the entire surface of the pupal body, except for the elytra and intersegmental membrane of the abdomen. In addition to the trichoid sensilla, almost all parts of the pupal body, including the elytra and the intersegmental membrane, had campaniform sensilla (CS) with a dome-like structure in the cuticular layer; these sensilla detected strains in the cuticle. The CS were concentrated on the articulation of segmented appendages and the lateral region of the intersegmental membrane. The defensive response (abdominal rotation) to electrical stimulation was greatly reduced when afferent nerves from CS clustered on the apical region of the tarsus were transected.

Conclusion: CS may be responsible for the high sensitivity of pupal appendages to tactile stimuli. The CS located on the appendages and abdominal intersegmental membrane may trigger specific and effective defensive behaviors. Both types of mechanoreceptive sensilla may enable pupal perception of the external environment, including the location of potentially harmful stimuli, and are involved in the control of defensive posture and movement.

Keywords: Coleoptera, pupa, defense, appendage, trichoid sensilla, campaniform sensilla

\section{Introduction}

The pupal stage is a vulnerable phase in the life of holometabolous insects. The pupae are usually covered with a hardened cuticle and protected within a cocoon or cell, ${ }^{1}$ while some species of beetles use chemical defense. ${ }^{2,3}$ Although most insect pupae are quiescent, some use persistent larval abdominal muscles to produce defensive responses. Hinton 4 coined the term "gin-trap" to describe the pinching device consisting of a pair of coapted, sclerotized jaws that are found in intersegmental regions of the abdomen of Coleopteran and Lepidopteran pupae. Tactile stimulation of the special region near the gin-trap triggers rapid and powerful closure of the jaws to damage small potential predators. ${ }^{5}$ In addition to the gin-trap closure response, pupae of the
Correspondence: Toshio Ichikawa Department of Biology, Faculty of Sciences, Kyushu University, Fukuoka 8I2-858I, Japan

Tel +81926422612

Fax +81926422315

Email tichiscb@kyuhsu-u.org 
tenebrionid beetle, Tenebrio molitor, exhibit another reflex behavior, ie, circular rotation of the abdominal segments in response to tactile stimulation of body appendages, including the legs, antennae, palps, and wings. ${ }^{6,7}$ This response is effective in protecting the pupa from larval cannibalism. ${ }^{8}$ However, little is known about the mechanosensory mechanisms that initiate these defensive behaviors.

The integuments of most insects are covered by many cuticular mechanoreceptive sensilla (mechanosensilla). The external structure of a mechanosensillum usually consists of a cuticular apparatus and a socket structure supporting the cuticular apparatus. ${ }^{7}$ The cuticular apparatus is specialized for detecting mechanical stimuli. The mechanosensilla are roughly categorized into hair-type and campaniform sensilla (CS). The hair-type sensilla consist of a cuticular apparatus protruding from the body surface, a socket, a receptor cell, a few supporting cells, and a glial cell. Hair sensilla respond to direct touch, faint air currents, low-frequency sound, and medium vibration. ${ }^{9}$ The CS are characterized by the presence of a dome-shaped cap (cupola) instead of a hair shaft. The cap is inserted through a modified joint membrane into a socket. The CS occur in many parts of the cuticle, especially in areas where mechanical stress exerted on the skeleton can cause deformation of their cupolas. Therefore, they mainly function as a proprioceptive sense organ that monitors external forces acting on the cuticle, or relative movements of segmental units, such as various cephalic and thoracic appendages (palp, antenna, wing, halter, and leg). ${ }^{10-12}$ The CS appear to perform similar functions in the abdominal segments of the bug, ${ }^{13}$ termite, ${ }^{14}$ locust, ${ }^{15}$ and the thoracic and abdominal segments of fly larvae. ${ }^{16,17}$

The pupa of Zophobas atratus is approximately ten times larger than that of T. molitor, and the former is suitable for morphological, behavioral, and physiological studies. ${ }^{8,18,19}$ To clarify the mechanosensory mechanism initiating pupal defensive behaviors, we examined the morphological distribution of mechanosensitive sensilla over the pupal body surface of $Z$. atratus. Because mechanical stress on the pupal cuticle appeared to be the most effective to induce pupal defensive behaviors, we performed physiological tests to determine whether the CS dominate these behaviors.

\section{Materials and methods}

\section{Animals}

Giant mealworms (Z. atratus Fab) were purchased as completely grown larvae from a local supplier. The detritivorous or omnivorous larvae were maintained under crowded conditions in a mixture of peat moss and sawdust and were fed fresh
Japanese radishes. Individual larvae were isolated in a plastic cup for pupation. The pupae were maintained at $26^{\circ} \mathrm{C} \pm 1^{\circ} \mathrm{C}$ under a 16-hour light/8-hour dark photoperiod for an average pupal period of 13 days. Pupal weight ranged from $0.4 \mathrm{~g}$ to $1.5 \mathrm{~g}$. To reduce interindividual variability in the number of mechanoreceptive sensilla, pupae of 0.6-1.0 g were selected for morphological examination. Individual appendages or parts from at least five pupae were examined.

\section{Scanning electron microscopy}

The abdomen of a pupa was injected with $0.3-1.0 \mathrm{~mL}$ of neutralized $10 \%$ formalin and fixed for 1-2 hours at room temperature. Individual appendages were isolated from the pupal body. The pupal abdomen was cut in half longitudinally along the medial plane of the abdomen. Adult antennae were excised from a beetle one day after adult ecdysis. All specimens were dehydrated in acetone. They were sonicated for 10 minutes and rinsed several times, then air-dried at room temperature and mounted on stubs. The mounted specimens were coated with a thin layer of gold in a sputtering unit and examined by scanning electron microscopy (Hitachi S-3000H).

\section{Transmission light microscopy}

Sensory cells, including mechanoreceptor cells in the appendage, were visualized with methylene blue. Methylene blue was dissolved in $2 \%$ formaldehyde neutral buffer solution, and $10-50 \mu \mathrm{L}$ of $0.75 \%$ methylene blue solution was injected into the thorax of a pupa. The pupa was kept at room temperature for 2-6 hours until the dye spread into the thoracic appendages (legs and elytra) via the hemolymph circulatory mechanism. ${ }^{17}$ To introduce the dye solution into cephalic appendages (antennae and maxillary palps), the solution was injected into the head capsule and distal parts of the appendages were punctured using a thin needle. After a small amount of dye-containing hemolymph was allowed to leak from the puncture, the pupa was kept at room temperature until the leak stopped naturally. An individual appendage was cut off from the base and mounted on a glass slide with an aqueous mounting medium and a glass coverslip. Specimens were examined with a transmission light microscope (Nikon E600).

Sensory cells in the pupal abdominal segments were visualized by injecting methylene blue solution into the abdomen. Distribution of the mechanosensilla (CS) in the abdominal segments was determined with $\mathrm{KOH}$-treated specimens. Tergites and sternites of a few abdominal segments were excised from a young pupa and immersed in $1 \% \mathrm{KOH}$ for 30 minutes. The specimens were washed several times in distilled water and remaining soft tissues were removed from 
the exoskeleton. Pupal cuticle peeled from the abdominal segments of an aged pupa (pharate adult) was also sometimes used for the examination. All specimens were dehydrated in a graded ethanol series, cleared in xylene, mounted on a glass slide, and examined by transmission light microscopy.

\section{Mechanical and electrical stimulations}

A one-day old pupa was placed ventral side up on a platform by fixing the dorsal part of its thorax to the platform with melted paraffin. Stimulation of a single sensory hair or group of hairs was performed manually with a piece of silver wire $(0.2 \mathrm{~mm}$ in diameter) under a binocular microscope. To test the sensitivity to mechanical forces over the entire pupal body, various parts were prodded with three probes of nylon filament or Nichrome wire $(0.2 \mathrm{~mm}$ in diameter $)$. Their bending forces, measured with a calibrated strain gage, were $0.6,6$, and $60 \mathrm{mN}$. The organs (or tissues) stimulated and their stimulation sites are listed in Table 1. Each probe was tested ten times at each stimulation site at an interval of 30 seconds.

To determine whether the CS on appendages play a significant role in the initiation of defensive behavior (abdominal rotation response), we performed two experiments. The first experiment tested response initiation by mechanical forces applied to the elytron surface. Because the elytron covers the hind wing and the hind leg femur, a mechanical force may bend the elytron to contact the underlying appendages. To block this contact, melted paraffin was poured under the elytra and hind legs on both sides. Then, the outer surface of the distal one-third of the right elytron was covered with a thin sheet of quick-drying epoxy glue,

Table I List of organs stimulated and their stimulation sites

\begin{tabular}{ll}
\hline Organs (tissues) & Stimulation sites \\
\hline Head & The frons \\
Antenna & 3rd flagellomere of the antenna \\
Maxillary palp & Anterior surface of the maxillary palp \\
Pronotum & Middle region of the pronotum \\
Leg (tarsus) & Apical portions of the tarsal segments of left \\
Leg (femur) & 3 legs \\
Elytron & Distal portions of femoral segments of left \\
Abdominal segment & 3 legs \\
(ventral) & Tip and middle region of the elytron \\
Abdominal segment & Ventral surface of the abdominal segment A3 \\
(dorsal) & Dorsal surface of the abdominal segment A3 \\
Intersegmental & \\
membrane (ventral) & Ventral region of the intersegmental \\
Intersegmental & membrane between A3 and A4 \\
membrane (dorsal) & Dorsal regions of the intersegmental \\
\hline
\end{tabular}

while that of the left elytron remained intact as a control. To estimate the sensitivity to mechanical forces, the tip of each elytron was stimulated 10 times with each of the three probes at an interval of 30 seconds. The second experiment tested response initiation by electrical stimulations of an appendage. After immobilizing a pupa by cooling it down in a refrigerator for 30 minutes, a small incision was made on the cuticle of the tarsal segment of the hind legs $0.5 \mathrm{~mm}$ away from the tip of the segment. The afferent nerve on the right leg was transected using microscissors to block sensory signals from the CS to the central nervous system, while the nerve on the left leg remained intact as a control. The incision was sealed with quick-drying glue. After the hind legs, excepting the tip of the tarsal segments, were immobilized with epoxy glue, two electrolytically sharpened tungsten needles (electrodes) mounted on a mechanical manipulator were gently inserted into the tip of a prepared tarsal segment. The electrodes were connected to a stimulator (Nihon-koden, Tokyo, Japan) and 10 bursts of $100 \mathrm{~Hz}$ pulses (50\% duty cycle) of different intensities (0.001-10 mA) were applied to the electrodes.

\section{Results \\ General morphology of pupal body}

The pupal body of $Z$. atratus consists of the head and three thoracic and nine abdominal segments; all the segmented parts of the body are slightly flattened in the dorsal-ventral axis. Spines or claw-like projections are aligned along the lateral flanges of the prothoracic and abdominal segments. All the thoracic appendages (legs and elytra) and the paired antennae are closely assembled into a complex structure that seems to augment their responsiveness to a mechanical stimulus. Each pupal leg consists of four segments corresponding to the imaginal coxa-trochanter complex, femur, tibia, and tarsus. The pupal tarsus has a partially segmented structure related to the imaginal tarsal segments. When the distal portion of the femoral segment of any leg in a one-day-old pupa was pushed gently with a force of $10 \mathrm{mN}$ $(1 \mathrm{~g})$, the segment usually bent $0.3-0.7 \mathrm{~mm}$. Thus, if a weak mechanical force was applied to an appendage, the force was easily transmitted to and stimulated adjacent appendages.

The mobile part of the pupal body is the abdominal segments, numbered A1-A9. A flexible intersegmental membrane connects adjacent abdominal segments. Abdominal segments A1-A6 have a narrow stripe (approximately $0.7 \mathrm{~mm}$ wide) of relatively thin, soft cuticle at the posterior marginal area of the sternite (or tergite). The cuticular zone can fold back to overlap the anterior marginal area of the 
next segment when the abdomen strongly bends, voluntarily or by an external force. Three claw-shaped spines are located on each lateral flange of segments A1-A7. The anterior and posterior spines are associated with a row of sclerotized teeth to form a jaw. The posterior jaw on one segment and the anterior jaw on the next segment make a pinching device, ie, the gin-trap. The third middle spine without teeth projects posterolaterally and is not a part of the pinching mechanism. Segment A8 has the third small spine only. Segment A9 has paired thorn-like processes (urogomphi) extending posteriorly. The basal part of the urogomphus is relatively elastic and the urogomphus is pliable around its base when an external force is applied to its sclerotized distal portion.

\section{Distribution of mechanoreceptive sensilla} Trichoid sensilla

Many hair (trichoid) sensilla of $80 \mu \mathrm{m}$ or more in length are present on the ventral and lateral surfaces of the pupal body (Figure 1). The bilateral distribution of the long sensilla is almost symmetrical, and most are located on the lateral area of each segmental part of the body. Longer hairs are situated on the vertex (four hairs), forehead (six hairs), mandible (3-4 hairs), labrum (2-4 hairs), maxillary palp (one hair), and antenna (four hairs). There is a row of 7-9 trichoid sensilla, each located beside a heavily sclerotized spine on the lateral flange of the prothorax (Figure 2A). Four sensilla appear on the lateral region of the prothorax. Each leg generally bears 10-20 sensilla distributed on the outer (ventral) surface of the femur and tibia (Figure 2B), while the inner (dorsal) surface of the leg has a few hairs. There is no hair on the elytron. The hind wing is almost completely covered with elytron and has two curved hairs (100-150 $\mu \mathrm{m}$ long) on the basal portion where the elytron can touch the hairs if pressed by a strong external force (data not shown).

The position of body surface hairs in the abdominal segments is serially homologous (Figure 1). Two pairs of hair sensilla curving posteriorly occur on each ventral sternite. Another sensillum extends posterolaterally from the base of the middle spine on each lateral flange of the abdominal segment.

Relatively short hairs (20-50 $\mu \mathrm{m}$ long) generally cover the entire pupal body, except for the elytra and the intersegmental areas of the abdomen. In addition to a dozen long hairs (80-150 $\mu \mathrm{m}$ in length) located in the marginal (anterior and lateral) areas of the femur of the foreleg, 10-20 short hairs are present in the posterior area of the femur (Figure 3 ). A few short hairs are usually seen on the dorsal surface of the tibia and the lateral surface of the tarsus. The pretarsus lacks hair sensilla. A similar distribution of the two classes of hair sensilla were observed in the middle and hind legs and the number of hairs was about two-thirds that of the forelegs.

A pupal antenna consists of the scape, pedicel, and nine flagellomeres. Trichoid sensilla (2-6 hairs, 50-125 $\mu \mathrm{m}$ long) are located on the distal region of each of the first to fifth flagellomeres (data not shown). The pupal antenna is characterized by hemispherical humps with a small pit on the top of the hump. A row of 13-15 humps are aligned along the distal border of the sixth to eighth flagellomeres, and 20-25 humps are scattered on the ventral surface of the ninth flagellomere (Figure 4A). The hump did not trigger a behavioral response to mechanical stimulation, thereby suggesting that the hump might be a precursor of the imaginal structure. Transmission photomicroscopic observation of the antennae of pharate adults often revealed a thick, imaginal sensillum beneath the hump of transparent pupal cuticle. Peeling old pupal cuticle from the pharate adult's antenna also revealed that thick sensilla (40 $\mu \mathrm{m}$ long and $10 \mu \mathrm{m}$ in basal width) were present near the humps (Figure 4B). The number and distribution of the imaginal sensilla were similar to those of the humps on the pupal cuticle.

The pupal maxilla consists of the cardo, stipes, lacinia, galea, and palp (Figure 5A). Two clusters of 10-20 hair sensilla were observed on the inner surface of the galea and lacinia (Figures 5B and 5C), which face the outer surface of the labrum with a narrow gap of $<50 \mu \mathrm{m}$. The galea usually has tiny hairs 3-6 $\mu \mathrm{m}$ long and one or two longer hairs. A large number of similar tiny hairs are arranged in a narrow strip along the ridge of the palp (data not shown). The hairs on the lacinia are relatively long $(20-60 \mu \mathrm{m})$ and thick, and are characterized by a strongly slanted posture (Figure 5C). The slanted hairs in the narrow gap may be stimulated indirectly if a mechanical force bends the maxillary palp medially.

Many short trichoid sensilla of 20-30 $\mu \mathrm{m}$ are scattered over major parts of individual abdominal segments, including the ventral and dorsal surface of the jaws and the urogomphi, at a density of approximately 5-20 hairs $/ \mathrm{mm}^{2}$, but they are few in the posterior marginal zone of the abdominal sternites and tergites. The intersegmental membrane lacks hair sensilla. Figure 6 shows a closeup view of a lateral region of the intersegmental membrane between abdominal segments A3 and A4. About 40 hair sensilla are discernible around the spiracle, but there are few on the wrinkled, intersegmental membrane, in spite of the fact that this particular area is 


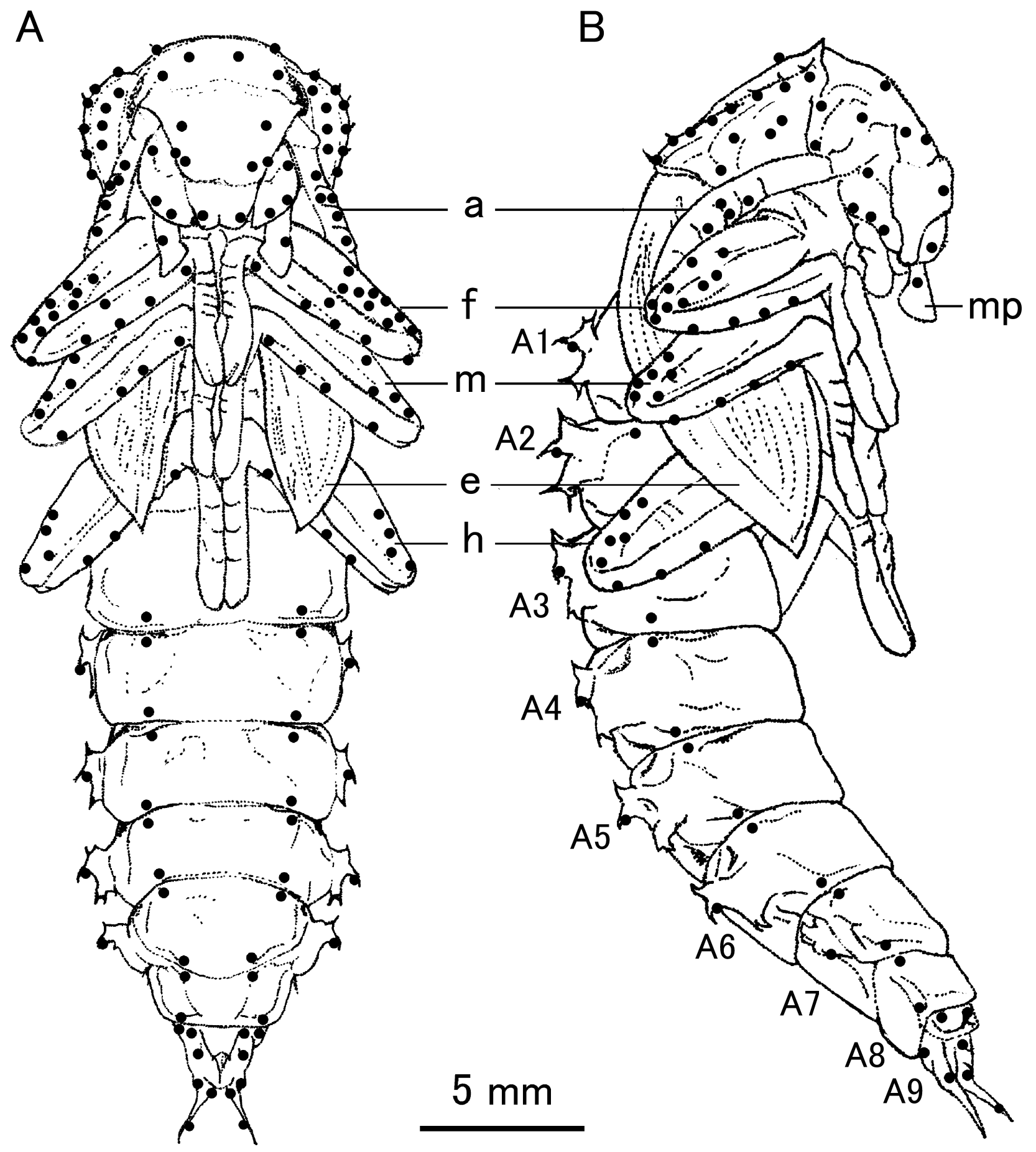

Figure I Distribution of longer hair sensilla $(\cdot)$ on the surface of the pupal body. Ventral (A) and lateral (B) aspects of a pupa. AI-A9, Ist to 9th abdominal segments. Abbreviations: a, antenna; $f$, foreleg; $m$, middle leg; e, elytron; $h$, hind leg; mp, maxillary palp

most sensitive to tactile stimulation and triggers the gin-trap closure response (Figure 5).

The short sensilla are also scattered on the surface of other parts of the pupal body, including the forehead (frons), vertex, labrum, labium, pronotum, mesothoracic tergum, and metathoracic tergum, at a density similar to that of the abdominal segments (data not shown). The elytron lacks short sensilla, while the hind wing has several short sensilla around the two long hair sensilla.

\section{Campaniform sensilla}

The hair-free areas of the pupal body, such as the distal portion of the tarsus (pretarsus), elytron, and intersegmental membrane of the abdomen, were highly sensitive to the tactile 

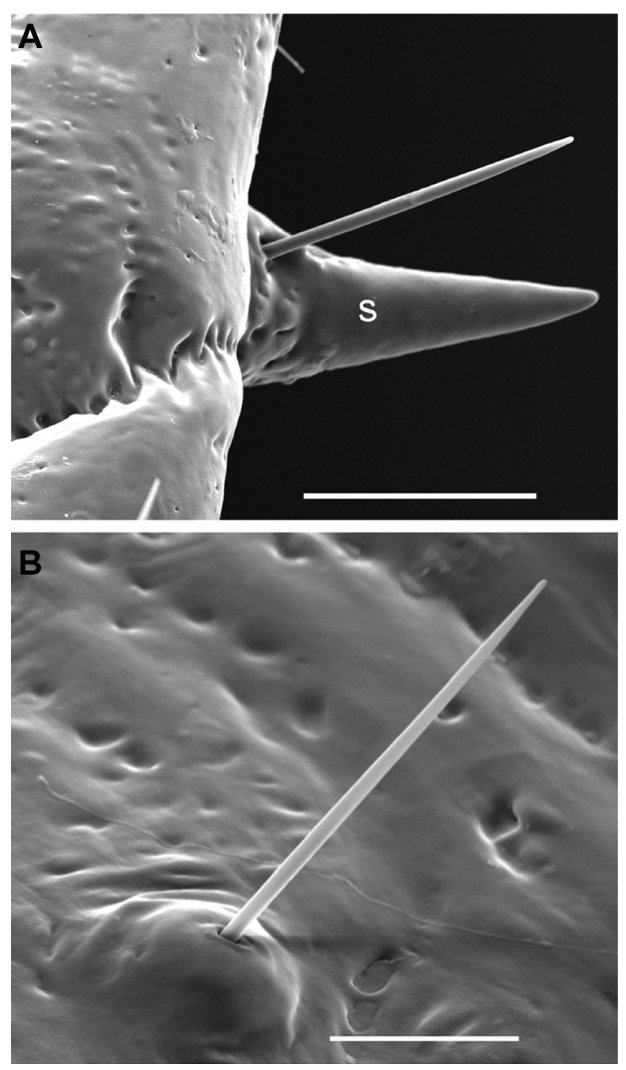

Figure $\mathbf{2}$ Long trichoid sensilla beside a sclerotized spine $\mathbf{A}$ ) and on the foreleg femur B). Scale bars, $100 \mu \mathrm{m} \mathrm{A)} \mathrm{and} 50 \mu \mathrm{m}$ B).

Abbreviation: s, spine.

stimulus and triggered a defensive response (Figure 6), suggesting that another type of mechanosensitive sensilla might function. Injection of methylene blue revealed thick nerve bundles, thin nerves branching from the nerve bundles, and distributed clusters of sensory cells (Figure 7).

Branching patterns of the thin nerves appeared to correspond to the segmental structures of individual appendages (Figure 7A). In the elytron, many clusters of cells were branched from seven bundles of nerves (Figure 7B). It was often discernible that each cell cluster contained 3-5 cell nuclei; one or a few of them might be sensory cells and the others were supporting cells. Neurites extending from individual cell clusters usually projected to the pupal cuticle, but most of them, especially in the antenna, terminated as a free ending in the cuticle. A small fraction of cell clusters extended neurites into the pupal trichoid sensilla and the hemispherical humps. In addition, some cell clusters extended a neural process into a cuticular apparatus embedded in the cuticle and appearing as a circular dome (7.5-8.5 $\mu \mathrm{m}$ in diameter) (Figure 8). The dome-shaped cuticular apparatus is a characteristic structure of the "campaniform" sensilla. The CS are most densely distributed on the tip of the tarsal segment, where $30-40$ sensilla are grouped into two clusters (Figures 8A and 8B). In other regions of the appendages, the $\mathrm{CS}$ were usually scattered singly or grouped loosely (Figure 8C). The cuticular apparatus of CS were distinguishable under a light microscope and their distribution over the appendages was examined (Figure 9). CS were often concentrated (3-15) on the articulation of segmented appendages (antenna, maxillary palp, and legs) where mechanical stress may be concentrated when a force is applied to the appendages. More than $150 \mathrm{CS}$ were observed on the ventral (outer) surface but not on the dorsal (inner) surface of the elytron. KOH-treatment revealed 75-100 CS on the ventral surface of the hind wing. In addition, 10-15 $\mathrm{CS}$ are located on the basal part of the wing together with a few haired sensilla; all others are aligned in a long, narrow strip along the midline of the wing.

Examination of CS distribution was extended to segmented parts of the pupal body. The abdominal intersegmental membrane (Figure 6) has many CS (Figure 8E); 60-80 CS are located in the lateral region of the intersegmental membrane, near the gin-trap, and most are anteriorly concentrated adjacent to the posterior edge of the sternite (Figure 10A). Abdominal tergites have two classes of CS, ie, large CS (10-12 $\mu \mathrm{m}$ in diameter) and small CS (6-8 $\mu \mathrm{m}$ in diameter). Large CS (80-90) are aligned along the anterior border of the hair-free, posterior marginal zone of each tergite of A1-A6, and many small CS are distributed on other areas of the tergite at a density of 10-40 sensilla/ $\mathrm{mm}^{2}$ (Figure 10B). The sternites of A1-A6 lack the large CS, and only small CS are distributed over almost the entire integument at a density of 5-45 sensilla/ $/ \mathrm{mm}^{2}$, except for the narrow, posterior marginal zone. Several CS are located around the base of claw-like projections (jaws) on the lateral flange of A1-A7. On the last three segments (A7-A9) that are hardened and tightly connected, there are more than $50 \mathrm{CS}$ along the posterior and lateral marginal areas. Thirty to $40 \mathrm{CS}$ are scattered on the surface of each urogomphus (Figure 10C).

In addition to $60-70 \mathrm{CS}$ aligned along the anterior margin of the pronotum (Figure 8D), there are 20-30 CS on the posterior marginal area of each thoracic segment. Approximately $60 \mathrm{CS}$ are distributed on the entire surface of the labrum and several CS are diffusely scattered on other parts of the head.

\section{Tactile sensitivity and function of campaniform sensilla}

Brushing of any appendage induced rapid rotation of abdominal segments in an all-or-none fashion. The direction 


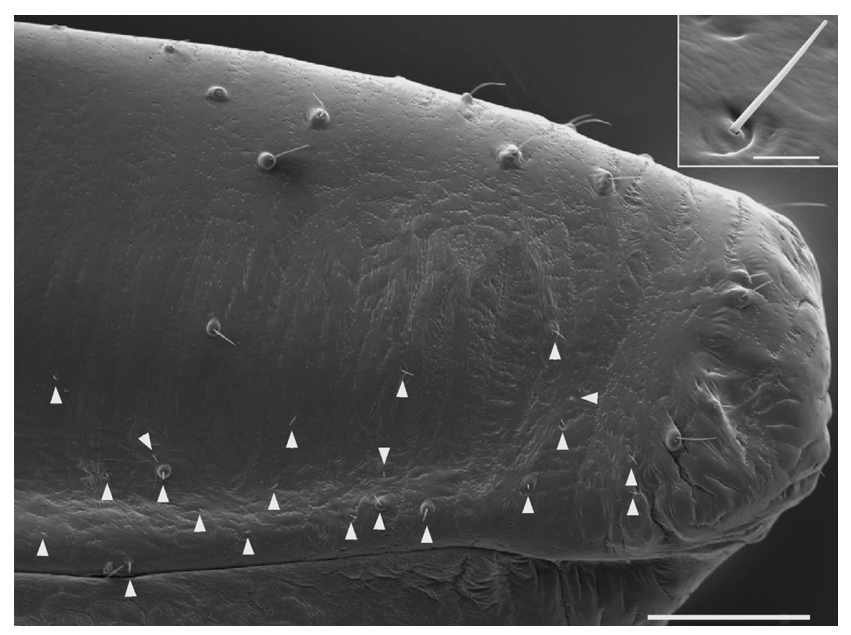

Figure 3 Ventral view of the lateral half of the foreleg femur. A majority of longer trichoid sensilla with a round socket occur on the anterior and lateral regions of the femur and some of them form a loose cluster. Many short hairs (arrowheads) occur on the posterior region. Inset, closeup view of a short hair. Scale bars, $0.5 \mathrm{~mm}, 20 \mu \mathrm{m}$ (inset).

of the abdominal rotation completely depended on the side of stimulation. Stimulation of a right (or left) appendage induced a right-handed (or left-handed) rotation about the anterior-posterior axis of the pupal body. Tactile stimulation of a single or a few hair sensilla on any appendage could induce abdominal rotation, but efficacy of the stimulation was usually low $(<30 \%)$. In contrast, prodding with a filament readily induced a response. To characterize the sensitivity profile of the entire pupal body, various parts were prodded with three probes of different stiffness (bending force of $0.6,6$, and $60 \mathrm{mN}$, Figure 11). Parts of body were classified by four levels of sensitivity. The most sensitive parts were the maxillary palp, the tip of the tarsal segment of three legs, and the elytron, all of which responded to a weak probe $(0.6 \mathrm{mN})$ with a high probability of $>80 \%$.
The antennae, femoral leg segments, ventral region of the intersegmental membrane, and the head capsule were less sensitive, responding to medium-intensity stimuli ( $6 \mathrm{mN})$ with $>80 \%$ probability. The dorsal region of the intersegmental membrane and ventral surface of the abdominal segments were far less sensitive, responding to strong stimuli $(60 \mathrm{mN})$ with a low probability $(15 \%-50 \%)$. The remaining regions, such as the dorsal surface of abdominal segments and the prothorax (pronotum), usually showed no response even to a strong stimulus, but they showed a significant response when they were strongly pinched with a pair of forceps (data not shown). Brushing of the lateral region of the intersegmental membrane near the gin-trap induced a gin-trap closure response, rather than abdominal rotation. The small, hair-free area between the posterior marginal zone (Figure 6) was most sensitive to the weak probe, and showed a gin-trap closure response at a high probability of $>80 \%$ (data not shown).

The result of the behavioral experiments did not always indicate that the defensive response was mediated by the CS in the appendages, because the mechanical force applied to an appendage might indirectly stimulate some hair sensilla of its own or those of a neighboring appendage. To understand whether the CS is important in the defensive response, CS responsiveness to a mechanical force or an electrical current was examined when transmission of the force to the sensilla or conduction of sensory signals from the sensilla to the central nervous system was hindered experimentally. The elytron and the tip of the tarsal segment having only CS were used as the site for stimulation. For the first experiment, the force needed to trigger a response at a probability of $50 \%$ was measured after the outer surface of the elytron
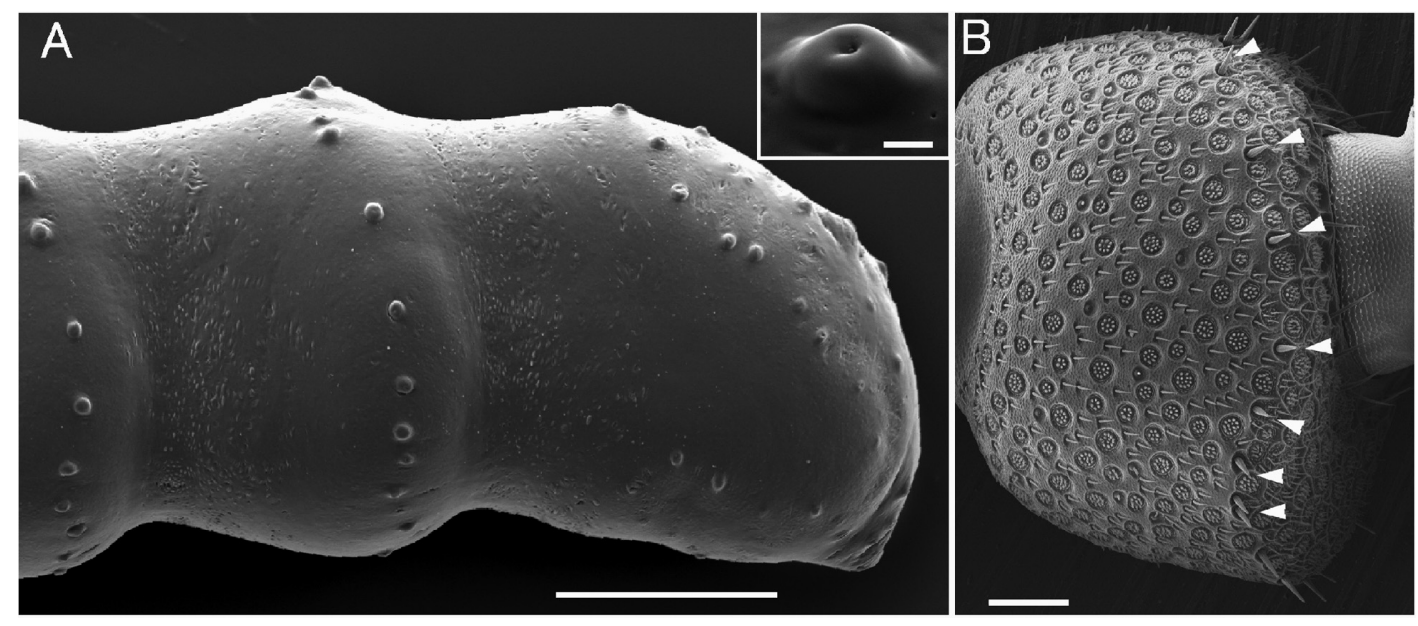

Figure 4 Ventral view of pupal A) and imaginal antenna B). A) Distal three flagellomeres (7th-9th) of the antenna. Many humps are seen. The inset shows a hump. Scale bars, $300 \mu$ m, $10 \mu \mathrm{m}$ (inset). B) 8th flagellomere of the adult antenna. A dozen thick hair sensilla (arrowheads) are seen among many other types of sensilla. Scale bar, $100 \mu \mathrm{m}$. 


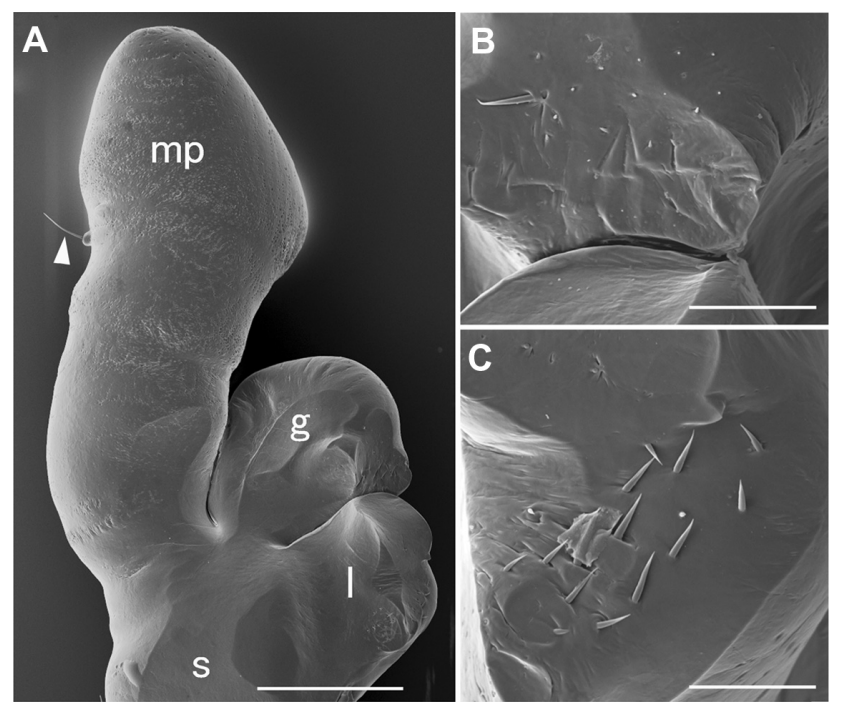

Figure 5 Medial view of the maxilla. A) long hair (arrowhead) is seen on the lateral surface of the maxillary palp, 17 short hairs and one longer hair on the galea $\mathbf{B}$ ) and 13 longer, slanted hairs and three short hairs on the lacinia $\mathbf{C}$ ) are seen. Scale bars, $0.5 \mathrm{~mm} \mathrm{~A}), 100 \mu \mathrm{m}$ (B, C).

Abbreviations: g, galea; I, lacinia; s, stipes; mp, maxillary palp.

on the right side was covered with a thin layer of epoxy glue. The force was $12.1 \pm 3.1 \mathrm{mN}(\mathrm{n}=6)$ for the covered elytron and $0.54 \pm 0.08 \mathrm{mN}(\mathrm{n}=6)$ for the intact elytron. Thus, an approximately 20 -fold (1.3 log units) stronger stimulus was required to induce a behavioral response, when the transmission of force to the surface was greatly reduced. For the second experiment, the CS localized on the tip of

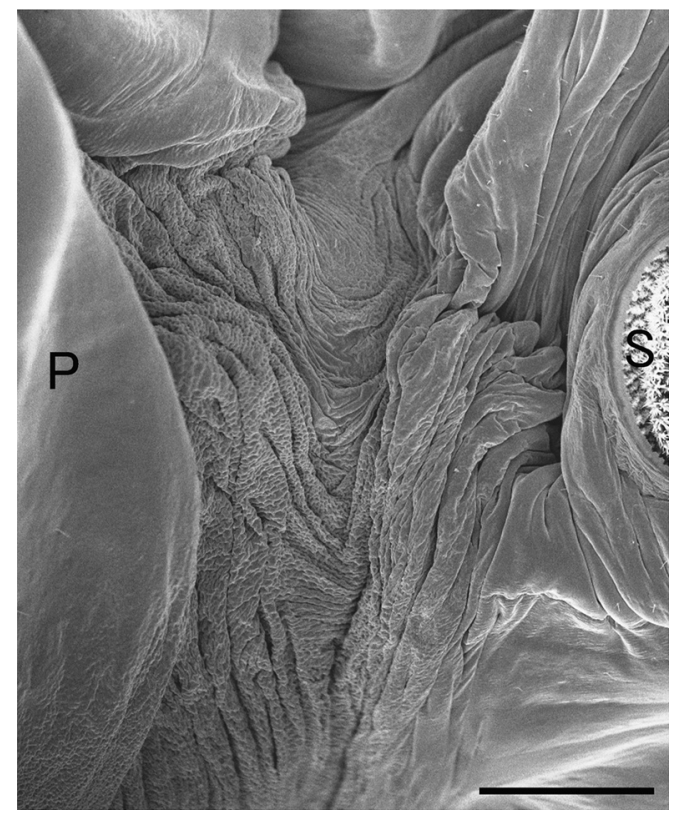

Figure 6 Lack of trichoid hairs in the intersegmental membrane of the abdomen. Lateral view of the lateral region of the intersegmental membrane between the posterior margin (P) of segment A3 and the spiracle (S) of A4. Approximately 40 hair sensilla are discernible around the spiracle, but they are absent in the wrinkled membrane. Scale bar, $200 \mu \mathrm{m}$. the hind leg were stimulated by electrical current pulses of different intensities after afferent nerves from the tarsal clustered CS on the right leg were surgically transected while those on the left leg remained intact. When electrical current pulses of $0.01 \mathrm{~mA}$ were applied to the control leg, responses were triggered at an efficiency of $>50 \%$ in all 10 tested pupae. In contrast, current pulses of $10 \mathrm{~mA}$ did not induce any defensive response when applied to the leg with a transected nerve. This result strongly suggests that CS on the appendages may be responsible for their high sensitivity to tactile stimuli and play a significant role in triggering defensive behavior.

\section{Discussion}

Pupae of the tenebrionid beetle $Z$. atratus bear mechanoreceptive hair sensilla on almost all parts of their body, except for the elytra and particular regions of the abdominal segment. The distribution of the sensilla was generally similar to that in another species of tenebrionid beetle, T. molitor ${ }^{20}$ Our results suggest that the hair sensilla may not generate the primary signal that activates neural mechanisms for pupal defensive responses (abdominal rotation and gin-trap closure), because stimulation of one or a few long hair sensilla on the appendages of $Z$. atratus induced abdominal rotation but the efficacy of the stimulation was low, the most sensitive part of the intersegmental membrane for triggering the gin-trap closure response is hair-free (Figure 6), and electrical stimulation of the tarsal CS readily induced the rotational response. However, we found that $Z$. atratus pupae exhibit a third complex defensive behavior consisting of rapid vibrations of the pupal body and irregular swings and rotations of the abdominal segments. The most effective stimulus for the activation of this irritable behavior was gentle brushing of the ventral surface of the abdominal segments (Ichikawa, unpublished observation). Thus, the short hair sensilla as well as the CS on the ventral surface of abdominal segments may be responsible for activation of the behavior. Many other hair sensilla may not be largely involved in triggering defensive behavior, but they may perceive mechanical information for the recognition of external environments, including potential enemies, and increasing arousal level for defensive behaviors.

This study revealed that almost all parts of the pupal cuticle, including the hair-free areas, have CS that could encode mechanical strains in the exoskeleton. ${ }^{9-12}$ Because the mechanical strain is not restricted to the local area of the cuticle pressed directly by a force and spreads widely through the cuticle to activate many strain sensors, CS may be useful as a mechanical sensor to detect a weak tactile stimulus. 


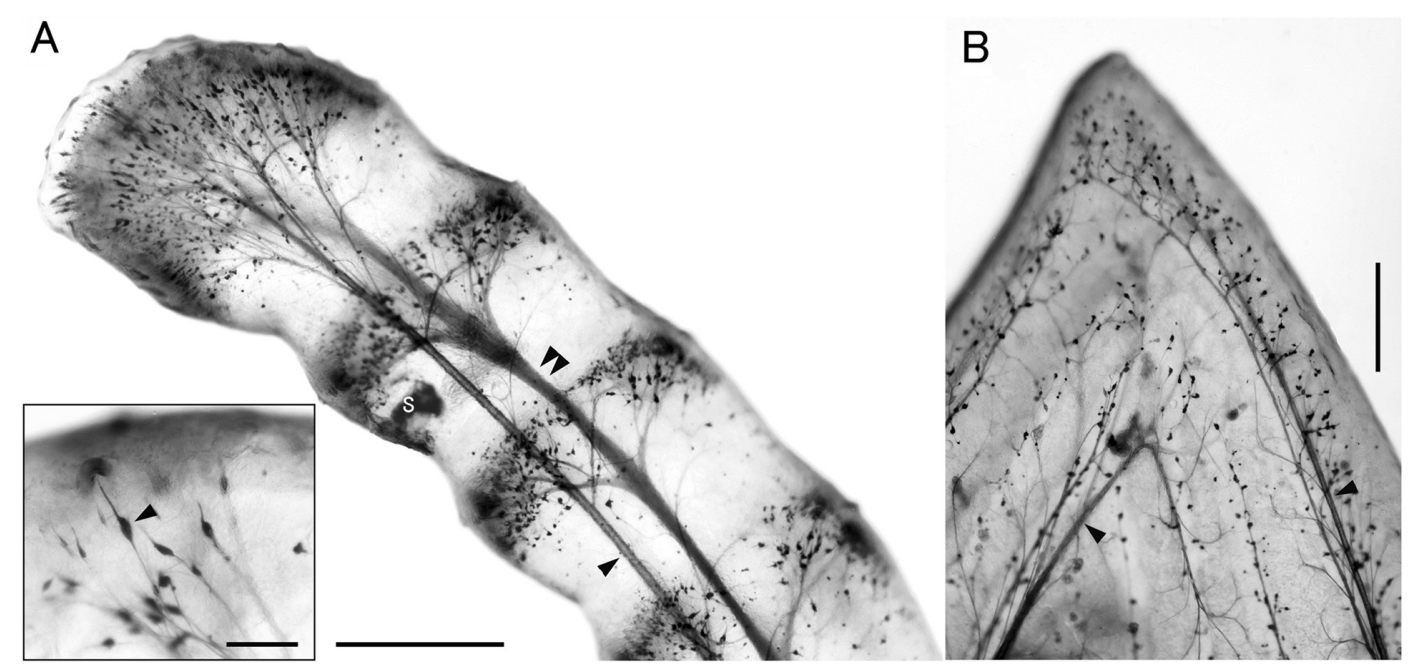

Figure 7 Distribution of sensory neurons stained with methylene blue in the distal three flagellomeres of the antenna $\mathbf{A}$ ) and an apical portion of the elytron B). Single arrowheads, trachea; double arrowhead, nerve bundle; s, scar of a puncture that drained dye-containing hemolymph. Scale bars, $500 \mu \mathrm{m}$ A), $200 \mu \mathrm{m}$ B). The inset shows a close-up view of an apical portion of the antenna. The arrowhead shows the sensory neuron projecting its dendrite to a hump. Scale bar, $50 \mu \mathrm{m}$.

The majority of insect CS have been identified by their characteristic external structures which are detectable by scanning electron microscopy., ${ }^{9,21,22}$ However, scanning electron microscopy in this study did not reveal the presence of CS on the pupal cuticle. A large number of CS are distributed on particular regions of the segmented parts of the pupal body (appendages and thoracic and abdominal segments) where the mechanical stress in the cuticle seemed to be concentrated when an external force was applied (Figures 8-10). An abdominal segment has many CS (Figure 10B) but mechanical stimulation induced very little defensive response
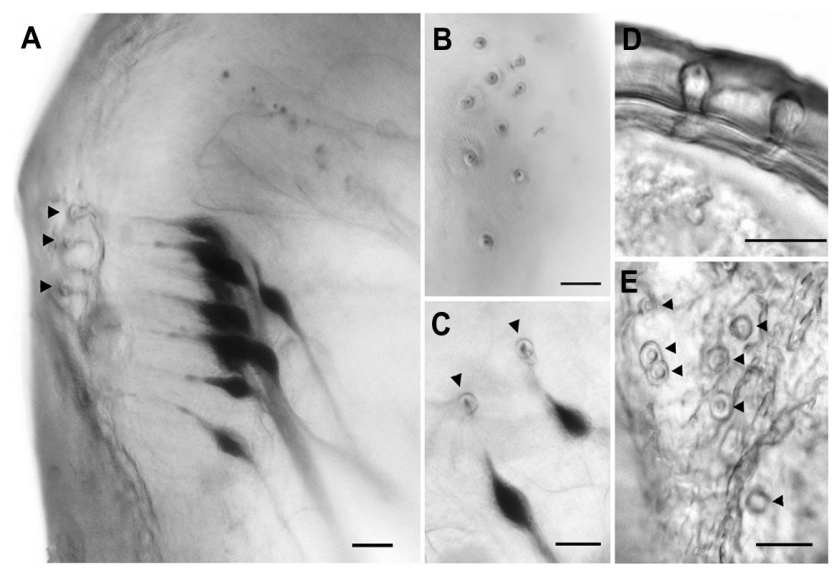

Figure 8 Transmission light micrographs of campaniform sensilla (CS) in various parts of the pupal body. Each arrowhead indicates the dome-like structure of a sensillum embedded in the cuticular layer. A) Lateral view of clustered CS in the tip of the hind leg tarsal segment. Somata of sensory cells and supporting cells were densely stained with methylene blue. B) Surface view of the clustered CS shown in A). Ten of 16 CS are visible in this focal plane. C) CS in the femur. A) distal end of the dendrite extending from a receptor cell is seen as a dot in the center of the cuticular dome. D) Lateral view of the cuticular apparatus of two CS embedded in the thick cuticle of the anterior edge of the pronotum. E) Surface view of the lateral area of the intersegmental membrane peeled from a pharate adult. Scale bars: $20 \mu \mathrm{m}$.
(Figure 11), suggesting that activation of some CS may not always produce a response. The likelihood of response may depend on the CS location, density, and mechanical sensitivity, which largely depends on the physical property of the pupal cuticle in which the CS is embedded. CS on different parts of the pupal body may have different physiological properties and functions.

The pupal appendages of tenebrionid beetles are exposed, immobile, and often the first target of cannibals ${ }^{8}$ and other potential predators. Thus, the CS located on the appendages (and the labrum) function as mechanical sensors that monitor harmful external stimuli and directly trigger a defensive response. The assembled structure of pupal legs, elytra, and antennae may facilitate mutual contact and increase the sensitivity to mechanical stimuli. The CS located on the specific lateral region of the intersegmental membrane near the gin-trap (Figure 10A) may also serve as the major mechanoreceptors that trigger another defensive response. The sensory and neuronal mechanisms of the pupal gin-trap closure responses have been intensively studied in hawkmoths, which have three pairs of gin-traps, each of which is a sharp-edged cuticular pit containing approximately 20 small mechanosensory hairs. ${ }^{23-25}$ Rapid closure of the gin-trap from the resting position is triggered by the stimulation of one or several mechanosensory hairs. A few CS are found in the cuticle between the trap and the hinge, but they do not mediate the sensory input for the response. ${ }^{23}$

The abdominal segments are the only mobile part of the pupal body, and many CS on the segments may function as proprioceptors monitoring the posture and movement 


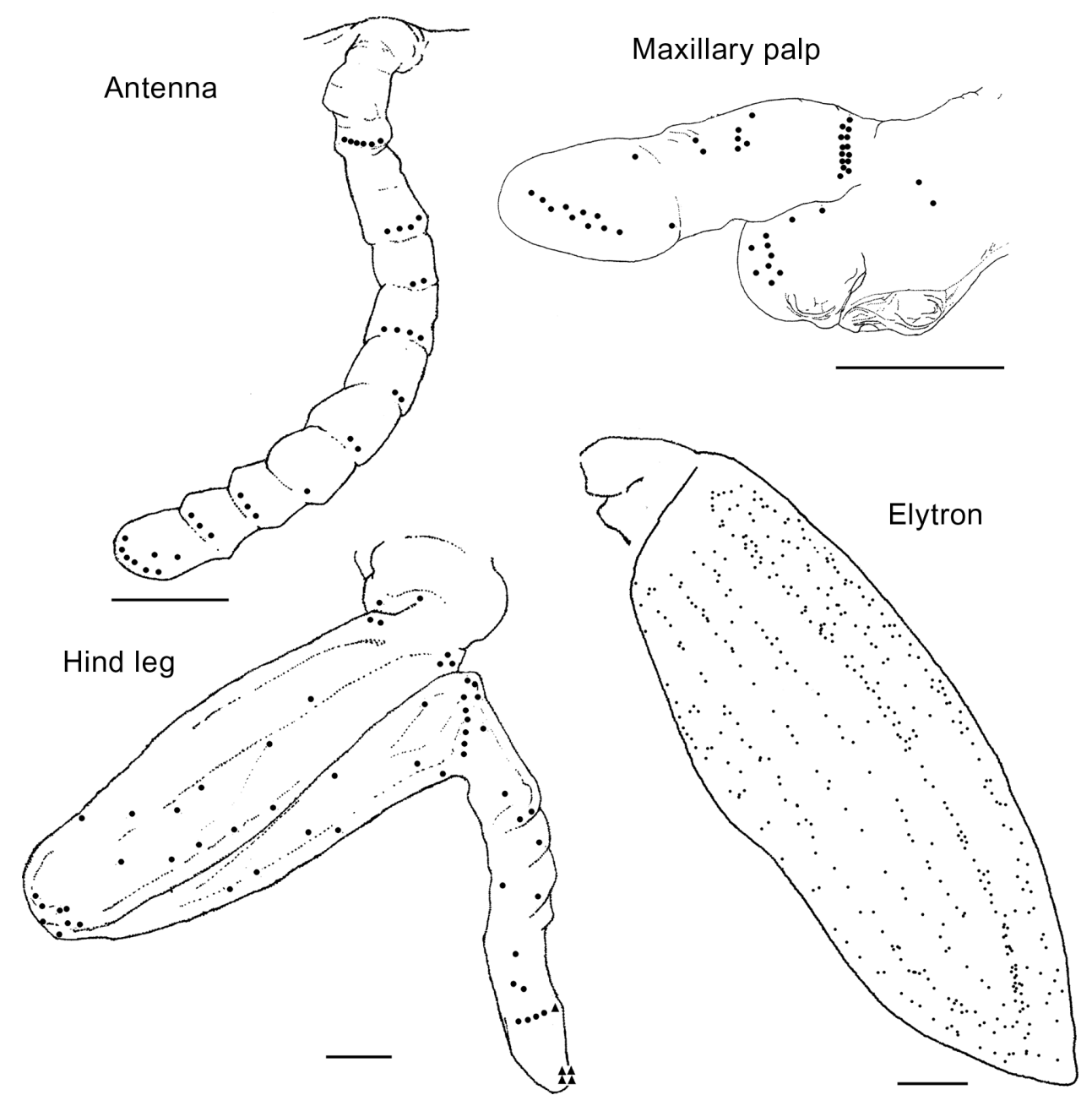

Figure 9 Distribution of campaniform sensilla (CS) on the ventral surface of appendages. Each dot indicates a single CS and each triangle indicates a cluster of 5 CS. Approximately $400 \mathrm{CS}$ were counted on the elytron. Scale bars, I $\mathrm{mm}$.

of the segment. The cuticle of the posterior marginal zone of each segment is relatively flexible and can bend or twist with abdominal movement; the CS aligned in the anterior border of the zone (Figure 10B) may serve as the proprioceptor. The last three abdominal segments (A7-A9) with paired urogomphi are tightly connected and bear many CS (Figure 10C). When abdominal rotation was induced in a freely lying pupa by tactile stimulation of an appendage, the rotation often made the pupa turn its dorsum to the source of stimulation. High-speed photography of the turn revealed that the mobile segments (A1-A6) transform the power of abdominal rotation into a $90^{\circ}$ turn of the pupal body around the longitudinal axis and that the thick urogomphi supported by the rigid segments serve as the fulcrum (Nakamura, unpublished observation). To perform such a rapid turn and any other abdomen-driven displacement of the pupal body successfully, peripheral sensory information concerning the degree of deformation in individual segments and the forces acting on the urogomphi may be important to control the central motor pattern. CS scattered in the abdominal segments were observed in developmental studies of sensory neurons and organs in larvae of the blood-sucking bug Rhodnius prolixus,,${ }^{13}$ blowfly,${ }^{16}$ and Drosophila.${ }^{17}$ The distribution of CS in the larval insects suggest that they may monitor the extension of individual segments during locomotion or blood sucking.

Because the pupa is a transitional stage from larva to adult, the pupal sensory system contains persisting larval neurons and newly differentiated or differentiating imaginal neurons. Studies on metamorphic changes in the central projections of hair sensilla on the body surface of T. molitor have shown that basic somatotopic organization of the projections in the larva is maintained in the adult, in spite of a profound reorganization of the body surface sensilla during 
A

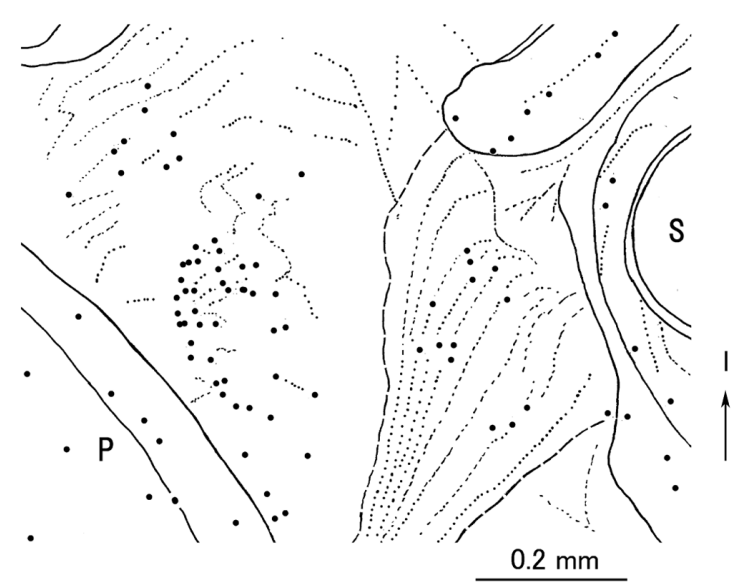

B

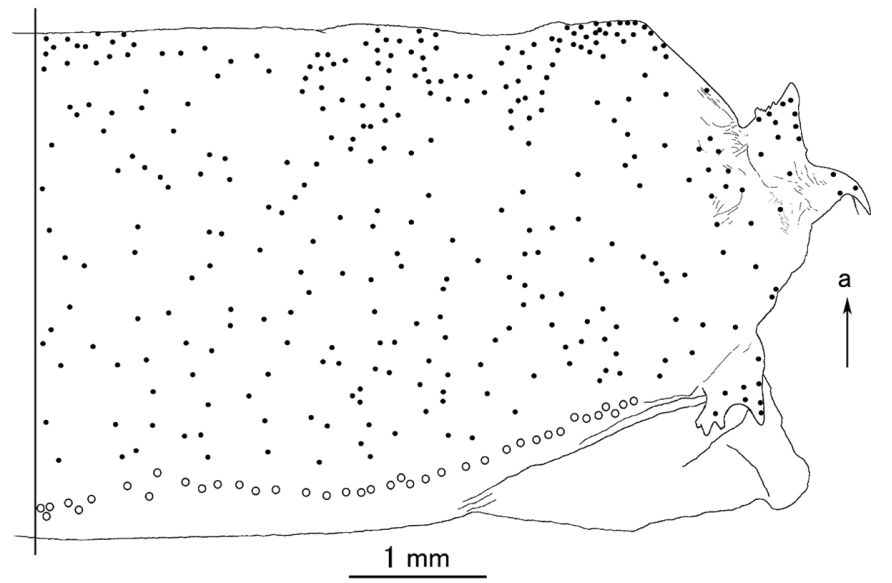

C

Dorsal

Ventral
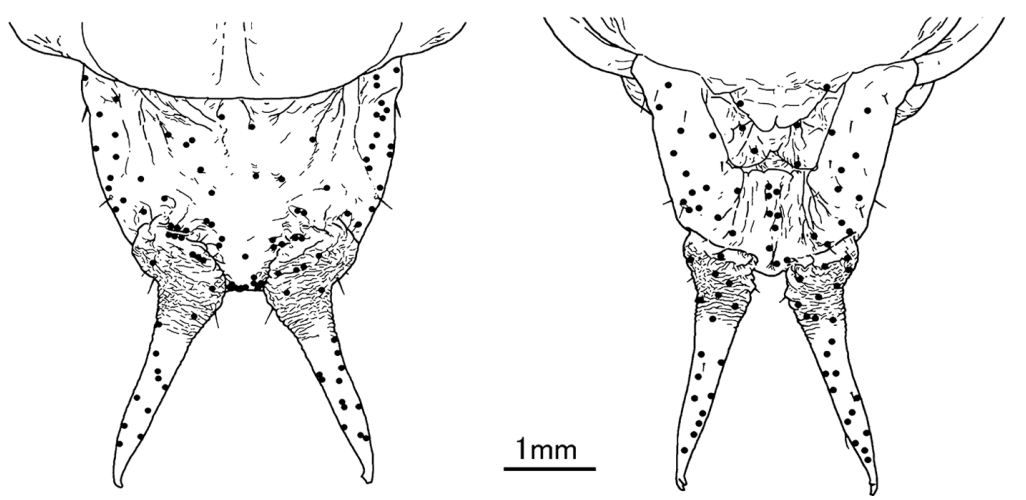

Figure 10 Distribution of campaniform sensilla (CS) in abdominal segments. A) Lateral region of the intersegmental region between abdominal segments A3 and A4. This region closely corresponds to the scanning electron microscopy image in Figure 6, but the pupal cuticle of a pharate adult is maximally extended to map the sensilla. Each dot indicates a single CS. B) Right half segment of the tergite of A4. Each circle and dot indicates a single large and small CS. C) 9th segment with a pair of urogomphi.

Abbreviations: $P$, posterior marginal area of $A 3$; $S$, spiracle of $A 4$; I, lateral; a, anterior.

metamorphosis..$^{20}$ The result suggests that many larval neurons may persist into the adult stage and/or at least into the pupal stage to act as a scaffold for growing imaginal sensory neurons. Typical persistent larval sensory neurons in the pupa of $Z$. atratus may be those that supply the majority of short hair sensilla on the abdominal segments, because similar hairs are often found on the corresponding areas of the larval abdomen at a similar density (Nakamura, unpublished observation). Because the elytron in the Coleoptera is derived from an imaginal disk-like precursor that is newly formed during postembryonic development, ${ }^{27}$ the pupal sensory neurons supplying CS on the beetle elytron likely differentiate at the larval-pupal transition. The majority of sensory neurons supplying the trichoid sensilla and CS on other pupal appendages (eg, Figures 3, 8, and 9) may be similar newly generated neurons, because morphological structures and sensory systems of the larval appendages (antennae and legs) in the tenebrionid beetles are significantly different from those of the pupal appendages. ${ }^{20,28}$ There were many (sensory) cells whose neurites terminated as free endings with no special cuticular apparatus on the pupal antenna (Figure 7A). The cells are possibly differentiating imaginal neurons that become functional after adult emergence and innervate various types of sensilla on the adult antenna, as shown in Figure 4B. The cell extending its neurite to the antennal hump (Figure 7A) may be a similar neuron, although it remains possible that the cell can function as a pupal sensory receptor of another modality.

We showed here that the tenebrionid pupa has many CS on the major parts of its exoskeleton, and that CS might function as proprioceptors and detectors of noxious external stimuli. It is important to know whether 


\begin{tabular}{|l|c|c|c|}
\hline \multicolumn{1}{|c|}{ Stimulation site } & & Force & \\
\hline & $0.6 \mathrm{mN}$ & $6 \mathrm{mN}$ & \\
\hline Maxillary palp & & & \\
\hline Leg (tarsus) & & & \\
\hline Elytron & & & \\
\hline Antenna & & & \\
\hline Leg (femur) & & & \\
\hline Int. membrane (ventral) & & & \\
\hline Head & & & \\
\hline Int. membrane (dorsal) & & & \\
\hline abd. segment (ventral) & - & & \\
\hline Pronotum & & & \\
\hline abd. segment (dorsal) & - & & \\
\hline
\end{tabular}

Figure II Profile of the mechanical sensitivity over the entire pupal body $(n=5)$. Large and small circles indicate response probability of $80 \%-100 \%$ and $55 \%-60 \%$. Dots and bars indicate response probability of $15 \%-30 \%$ and $0 \%-5 \%$.

the strategy to distribute many CS on the body surface is specific to the pupal stage or common to the larval and adult stages. In a preliminary experiment, we found that larval thoracic and abdominal segments of $Z$. atratus and T. molitor had many CS in their hard cuticle and the distribution pattern of larval CS was similar to that of pupal CS, thereby suggesting that the beetles at the larval stage may continue to utilize CS as proprioceptors to monitor cuticular distortions produced by changes in the relative position and movement of the segments. It is also likely that some larval CS function as receptors or nociceptors that detect the location and strength of various kinds of mechanical stimuli, including harmful stimuli. This notion raises an important question: is the structural principle of the somatosensory system of hard-bodied larvae different from that of soft-bodied larvae such as $M$. sexta and Drosophila melanogaster? Drosophila larvae have a sensory network consisting of multiple classes of multidendritic neurons tiling all over their soft body walls to sense mechanical and thermal signals. ${ }^{29}$ The multidendritic neurons in Manduca larvae appear to function as proprioceptors and touch receptors (nociceptors), ${ }^{30}$ and seem to contribute to the selective triggering and sensitization of defensive reflexes by noxious stimuli. ${ }^{31}$ Similar proprioceptive and nociceptive functions of multidendritic neurons were reported in Drosophila larvae during locomotion and defensive (or nocifensive) behaviors. ${ }^{32,33}$ Recently, Xiang et al ${ }^{34}$ reported that the multidendritic neurons mediated an avoidance response to strong, harmful light stimuli. To answer our question, we must determine whether the hard-bodied larvae have a similar network of multidendritic neurons and must characterize the physiology of the larval CS.

\section{Disclosure}

The authors report no conflicts of interest in this work.

\section{References}

1. Hinton HE. Protective devices of endopterygote pupae. Trans Soc Brit Entomol. 1955;12:49-92.

2. Daloze D, Breakman JC, Pasteels JM. Ladybird defence alkaloids: Structural, chemotaxonomic and biosynthetic aspects (Col.: Coccinellidae). Chemoecology. 1994;5:173-183.

3. Schröder FC, Farmer JJ, Attygalle AB, Smedley SR, Eisner T, Meinwald J. Combinational chemistry in insects: A library of defensive macrocyclic polyamines. Science. 1998;281:428-431.

4. Hinton HE. The "gin-traps" of some beetle pupae; a protective device which appears to be unknown. Trans R Entomol Soc Lond. 1946;97: 473-496.

5. Wilson MCL. The morphology and mechanism of the pupal gin-trap of Tenebrio molitor L. (Coleoptera, Tenebrionidae). J Stored Prod Res. 1971;7:21-30.

6. Hollis JH. Habituatory response decrement in pupae of Tenebrio molitor. Anim Behav. 1963;11:161-163.

7. Askew HR, Kurtz PJ. Studies on the function of the abdominal rotation response in pupae of Tenebrio molitor. Behaviour. 1974;50: 152-171.

8. Ichikawa T, Kurauchi T. Larval cannibalism and pupal defense against cannibalism in two species of tenebrionid beetles. Zool Sci. 2009; 26:525-529.

9. Keil TA. Functional morphology of insect mechanoreceptors. Micros Res Tech. 1997;39:506-531.

10. Pringle JSW. Proprioception in insects. I. A new type of mechanical receptor from the palps of the cockroach. J Exp Biol. 1938;15:101-113.

11. Spinola SM, Chapman KM. Proprioceptive indentation of the campaniform sensilla of cockroach legs. J Comp Physiol. 1975;96:257-272.

12. Zill SN, Moran DT. The exoskeleton and insect proprioception. 1. Responses of tibial campaniform sensilla to external and musclegenerated forces in the American cockroach, Periplaneta americana. J Exp Biol. 1981;91:1-24.

13. Wigglesworth VB. The origin of sensory neurones in an insect, Rhodnius prolixus (Hemiptera). Quart J Microsc Sci. 1953;94:93-112.

14. Stuart AM, Satir P. Morphological and functional aspects of an insect epidermal gland. J Cell Biol. 1968;36:527-549.

15. Tousson E, Hustert R. External sensilla of the locust abdomen provide the central nervous system with an interganglionic network. Cell Tissue Res. 2006;325:151-162.

16. Osborne MP. The sensory neurons and sensilla in the abdomen and thorax of the blowfly larva. Quart J Microsc Sci. 1963;104:227-241.

17. Dambly-Chaudière C, Ghysen A. The sense organs in the Drosophila larva and their relation to the embryonic pattern of sensory neurons. Roux's Arch Dev Biol. 1986;195:222-228. 
18. Ichikawa T. Periodic abdominal pumping supports leg development during metamorphosis in tenebrionid beetle Zophobas atratus. Comp Biochem Physiol A. 2008;150:8-13.

19. Ichikawa T. Mechanism of hemolymph circulation in the pupal leg of tenebrionid beetle Zophobas atratus. Comp Biochem Physiol A. 2009; 153:174-180.

20. Breidbach O. Metamorphic changes in the central projections of hair sensilla in Tenebrio molitor L. (Insecta: Coleoptera). Cell Tissue Res. 1990;259:159-176.

21. Toh Y. Fine structure of sense organs on the antennal pedicel and scape of the male cockroach, Periplaneta Americana. JUltrastruct Res. 1981; 77:119-132.

22. Grünert U, Gnatzy W. Campaniform sensilla of Calliphora vicina (Insecta, Diptera) II. Typology. Zoomorphology. 1987;106:320-328.

23. Bate CM. The mechanism of the pupal gin trap. I. Segmental gradients and the connexions of the triggering sensilla. J Exp Biol. 1973; 59:95-107.

24. Levine RB, Truman JW. Peptide activation of a simple neural circuit. Brain Res. 1983;279:335-338.

25. Waldrop B, Levine RB. Development of the gin trap reflex in Manduca: A comparison of larval and pupal motor responses. J Comp Physiol A. 1989;165:743-753.

26. Lemon WC, Levine RB. Multisegmental motor activity in the segmentally restricted gin trap behaviour in Manduca sexta pupae. J Comp Physiol A. 1997;180:611-619.
27. Angelini DR, Kaufman TC. Insect appendages and comparative ontogenetics. Dev Biol. 2005;286:57-77.

28. Farazmand H, Chaika SY. Morphology and ultrastructure of the antennal sense organs in tenebrionid larvae Tenebrio molitor L. and Zophobas rugipes Kirsch (Coleoptera: Tenebrionidae). Moscow Univ Biol Sci Bull. 2007;62:76-82.

29. Grueber WB, Gruabard K, Truman JW. Tiling of the body wall by multidendritic sensory neurons in Manduca sexta. J Comp Neurol. 2001;440:271-283.

30. Grueber WB, Lan LY, Yan YN. Tiling of the Drosophila epidermis by multidendritic sensory neurons. Development. 2002;129:2867-2878.

31. Walters ET, Illich PA, Weeks JC. Defensive responses of larval Manduca sexta and their sensitization by noxious stimuli in the laboratory and fields. J Exp Biol. 2001;204:457-469.

32. Song W, Onishi M, Jan LY, Jan YN. Peripheral multidendritic sensory neurons are necessary for rhythmic locomotion behavior in Drosophila larvae. Proc Natl Acad Sci U S A. 2007;104:5199-5204.

33. Hwang RY, Zhong L, XuY, et al. Nociceptive neurons protect Drosophila larvae from parasitoid wasps. Curr Biol. 2007;17:2105-2116.

34. Xiang Y, Yuan Q, Vogt N, et al. Light-avoidance-mediating photoreceptors tile the Drosophila larval body wall. Nature. 2010;468:921-926.

Open Access Insect Physiology

\section{Publish your work in this journal}

Open Access Insect Physiology is an international, peer-reviewed, open access journal publishing original research, reports, reviews and commentaries on all areas of insect physiology. The manuscript management system is completely online and includes a very quick and fair peer-review system, which is all easy to use.

\section{Dovepress}

Visit http://www.dovepress.com/testimonials.php to read real quotes from published authors. 